\title{
Fabrication of Low-Cost Paper-Based Microfluidic Devices by Embossing or Cut-and-Stack Methods
}

\section{Citation}

Thuo, Martin M., Ramses V. Martinez, Wen-Jie Lan, Xinyu Liu, Jabulani Barber, Manza B. J. Atkinson, Dineth Bandarage, Jean-Francis Bloch, and George M. Whitesides. 2014. "Fabrication of Low-Cost Paper-Based Microfluidic Devices by Embossing or Cut-and-Stack Methods." Chem. Mater. 26 (14) (July 22): 4230-4237. doi:10.1021/cm501596s.

\section{Published Version}

doi: $10.1021 / \mathrm{cm} 501596 \mathrm{~s}$

\section{Permanent link}

http://nrs.harvard.edu/urn-3:HUL.InstRepos:25811019

\section{Terms of Use}

This article was downloaded from Harvard University's DASH repository, and is made available under the terms and conditions applicable to Open Access Policy Articles, as set forth at http:// nrs.harvard.edu/urn-3:HUL.InstRepos:dash.current.terms-of-use\#OAP

\section{Share Your Story}

The Harvard community has made this article openly available.

Please share how this access benefits you. Submit a story.

\section{Accessibility}




\section{Fabrication of Low-cost Paper-based Microfluidic}

\section{Devices by Embossing or Cut-and-Stack Methods}

Martin M. Thuo ${ }^{1 \dagger}$, Ramses V. Martinez ${ }^{1 \dagger}$, Wenjie Lan ${ }^{1}$, Xinyu Liu ${ }^{1}$, Jabulani Barber ${ }^{1}$, Manza

B.J. Atkinson ${ }^{1}$, Dineth Bandarage ${ }^{1}$, Jean-Francis Bloch ${ }^{1,2}$, and George M. Whitesides ${ }^{1,3 *}$

AUTHOR ADDRESS.

1. Department of Chemistry and Chemical Biology, Harvard University, 12 Oxford Street, Cambridge, MA 02138, USA.

2. Department of Papermaking Engineering - LGP2 Grenoble Institute of Technology, 461 rue de la Papeterie, BP65 - 38402 Saint Martin d'Hères, Cedex, France.

3. Wyss Institute for Biologically Inspired Engineering, Harvard University, 60 Oxford Street, Cambridge, MA 02138, USA.

* Corresponding authors E-mail: gwhitesides@gmwgroup.harvard.edu

$\dagger$ Authors contributed equally to this work.

KEYWORDS. Microfluidics, Omniphobic paper, Embossing, Cut-and-stack, Paper-based Microfluidics. 


\begin{abstract}
This communication describes the use of embossing, and "cut-and-stack" methods of assembly, to generate microfluidic devices from omniphobic paper, and demonstrates that fluid flowing through these devices behaves similarly to fluid in an open-channel microfluidic device. The porosity of the paper to gasses allows processes not possible in devices made using PDMS or other non-porous materials. Droplet generators and phase separators, for example, could be made by embossing " $T$ ”-shaped channels on paper. Vertical stacking of embossed or cut layers of omniphobic paper generated three-dimensional systems of microchannels. The gas permeability of the paper allowed fluid in the microchannel to contact and exchange with environmental or directed gases. An aqueous stream of water containing a $\mathrm{pH}$-indicator, as one demonstration, changed color upon exposure to air containing $\mathrm{HCl}$ or $\mathrm{NH}_{3}$ gases.
\end{abstract}


The most widely used technology for the formation of microfluidic systemsfabrication using polydimethylsiloxane (PDMS) and soft-lithography - is still too expensive and/or technically demanding for applications requiring low cost (e.g., human and veterinary medicine in resource-poor settings, ${ }^{1}$ food testing, ${ }^{2}$ or environmental monitoring ${ }^{3}$ ). To circumvent the issues of cost and availability, we, ${ }^{4-6}$ and others, ${ }^{7-9}$ have developed so-called "paper microfluidics" (a methodology in which fluid flow in paper is driven by capillary wetting, and directed by channel boundaries of hydrophobic wax ${ }^{10}$ or polymer patterned in the paper by printing, ${ }^{7}$ photolithography, ${ }^{11}$ or other methods ${ }^{12}$ ). Although wicking-driven paper devices are clearly attractive for their low cost, simplicity, and ability to generate 3D microfluidic systems, the cellulose fibers that partially fill the channels introduce complexity into the flow of liquids - a problem that is largely absent in open-channel PDMS-based microfluidic devices. These wicking-based devices also present a large area of potentially adsorptive surface (which may be useful or detrimental, depending on the application); the performance of wicking devices may also be influenced by environmental factors such as humidity.

This paper is one of several introducing a new technology that, among other things, enables paper to be used as the basis for pressure-driven open-channel microfluidic systems. ${ }^{13,14}$ The key element in this technology is “omniphobic paper": that is, paper whose surface has been modified by treatment with a highly fluorinated alkylsilane (and which we therefore call " $\left.\mathrm{R}^{\mathrm{F} \text { " paper }}{ }^{14}\right)$. The static contact angle $\left(\theta_{\mathrm{s}}\right)$ of water on $\mathrm{R}^{\mathrm{F}}$ paper is high, $\theta_{\mathrm{s}}=135^{\circ}-155^{\circ}\left(\right.$ greater than water on Teflon, $\left.\theta_{\mathrm{s}}=120^{\circ}\right) \cdot \mathrm{R}^{\mathrm{F}}$ paper is also able to resist wetting by many organic liquids: liquids with surface tensions as low as $27 \mathrm{mN} / \mathrm{m}$ (hexadecane) do not spread on $\mathrm{R}^{\mathrm{F}}$ paper. ${ }^{14}$ Paper can be readily formed into a variety of complex 3D shapes by embossing, engraving, cutting, folding, stacking, molding, or bending. The surface modification of these paper structures by silanization (or other chemistry) 
enables them to be used as microfluidic devices. Combination of the shaped, surfacemodified paper with other materials (polymer tapes, hydrophilic paper and thread, metal films, etc.) generates a wide range of functions and devices having low weight and low cost of materials. ${ }^{13}$ Shaped, $\mathrm{R}^{\mathrm{F}}$ paper also enables new functions, because it has properties not readily encountered in conventional materials (for example, it is simultaneously inexpensive, lightweight, highly hydrophobic, highly gas permeable, easily burned, and thermally/electrically insulating).

We have described one versatile technique that involves cutting channels in cardstock paper using a programmable knife, ${ }^{13}$ making these engraved structures omniphobic by silanizing them using a fluorinated alkyltrichlorosilane $\left(\mathrm{R}^{\mathrm{F}} \mathrm{SiCl}_{3}\right)$ in the vapor phase, and sealing the channels with pre-cut transparent tape. ${ }^{14}$ This paper describes a related method for fabricating simple open-channel microfluidic systems on Whatman ${ }^{\circledR} \# 1$ filter paper, based on embossing using reusable plastic molds or cutting and stacking appropriate structures into the filter paper followed by the silanization step previously mentioned. The former technique yields smaller channels, and is easier to carry out; The latter is more useful for larger features, and parallel production of large number of devices (by reel-to-reel embossing and processing).

Paper microfluidics, as a generic technology, would benefit from materials and systems that would preserve the advantages of paper while allowing fluids to flow in a pressure-driven open channel. These devices would find application in situations requiring the control of fluid flows afforded by open channels (for example, high-resolution capillary electrophoresis ${ }^{15}$ ), applications that require manipulation of fluids containing suspended particles (such as blood, environmental slurries, multi-phase suspensions, and most unprocessed biological samples), ${ }^{16,17}$ in analysis and/or manipulation of mixtures of 
compounds that would separate chromatographically in a wicking-based devices, ${ }^{18}$ or the manipulation of complex chemical mixtures. ${ }^{19,20}$ Open-channel microfluidic devices might also be useful in the synthesis of particles ${ }^{21,22}$ and in processes, such as microfluidic shear separation, ${ }^{23}$ where the fluidic-flow properties of a liquid are of interest.

The surfaces of the cellulose fibers that make up paper have a high density of hydroxyl and acetal groups, and are normally hydrophilic. Paper can be made hydrophobic by physisorption (coating the fibers or filling the voids with a hydrophobic material) ${ }^{7}, 10$ or chemisorptions (applying reactions that modify the $\mathrm{OH}$ groups, such as silanization, acylation, or epoxide and thiirane ring opening). ${ }^{12}$ Of these, we have chosen silanization because: i) the reaction occurs readily at room temperature when the hydroxyl groups are exposed to vapors of a silanizing agent and ii) there is a variety of commercially available organosilanes, with different terminal groups, that can be used to modify the surface of paper. Exposure of Whatman \#1 filter paper, for example, to vapors of many perfluorinated alkylsilanes, $\mathrm{R}^{\mathrm{F} S i C l} 3$, under vacuum renders it hydrophobic (static contact angle of water, $\left.\theta_{\mathrm{s}}>135^{\circ}\right) .{ }^{14}$ The reaction of the hydroxyl groups of paper and alkyltrichlorosilanes, $\mathrm{R}^{\mathrm{F}} \mathrm{SiCl}_{3}$, is rapid and requires no catalyst. This reagent does not react with acetal moieties, and as such, can be used for surface functionalization of paper without damaging the linkages that make up the backbone of the polymeric cellulose.

\section{Results and Discussion}

Fabrication of Dies for Embossing Paper. The polymeric dies used to emboss open channels on paper were designed using computer-aided design (CAD, Airlibre Inc.) and generated by 3D printing (StrataSys Dimension Elite) using acrylonitrile butadiene styrene (ABS) co-polymer. ${ }^{24}$ Paper releases readily from these ABS dies after embossing. We have 
used the dies over a hundred times with no damage or degradation in performance. Dies for embossing can also be generated via other processes such as thermoplastic casting, laser cutting, or selective etching. ${ }^{25}$ The supporting information (Figure S1 and S2) gives detailed schematics and pictures of the embossing dies we used.

Fabrication of Open-channel Microfluidic Devices by Embossing. We fabricated the open-channel microfluidic devices by sandwiching a sheet of paper between two dies with complimentary shapes (see Figure 1a-b). Paper is compressed into a channel by applying pressure $\left(\sim 0.2 \mathrm{~kg} / \mathrm{cm}^{2}\right)$. To increase moldability, we put a few drops of ethanol $(\sim 400 \mu \mathrm{L}$ per device) on the paper to wet its surface and reduce its glass transition temperature. ${ }^{26}$ Wetting the paper with liquid ethanol makes the paper easier to emboss with less force and avoids tearing the paper on the edges. Once embossing was finished $(\sim 2 \mathrm{~s})$, the paper was allowed to dry for $\sim 30 \mathrm{~s}$ in an oven at $60^{\circ} \mathrm{C}$.

Silanization of Paper Devices. We made the paper devices omniphobic by silanization with $\mathrm{R}^{\mathrm{F}} \mathrm{SiCl}_{3}$ (trichloro(1H,1H,2H,2H-perfluorooctyl)silane, $\left.\mathrm{CF}_{3}\left(\mathrm{CF}_{2}\right)_{5}(\mathrm{CH})_{2} \mathrm{SiCl}_{3}\right)$ (obtained from Sigma-Aldrich and used as supplied) in a solid-vapor reaction. ${ }^{14,27} \mathrm{R}^{\mathrm{F} S i C l} 3$ are relatively inexpensive: we used $\sim 1 \mathrm{~g}$ of reagent $(\$ 0.60)$ to functionalize a quantity of paper required to make $>100$ devices $\left(\sim 600 \mathrm{~cm}^{2}\right.$, or $<\$ 0.006 /$ device $)$.

Assembly of the Microfluidic Devices. We covered the channels with transparent adhesive tape (Fellowes adhesive sheet, PET/EVA/LDPE) on which inlet and outlet holes had been cut (using a laser cutter or a punch, see Figure 1c). This process for fabrication of devices is rapid (>100 devices/h) and requires no special facilities or tools. We employed this two-step method to fabricate microfluidic devices and multiple-well-plates. Figure $1 \mathrm{~d}$ and $1 \mathrm{e}$ 
show a 2D microfluidic device with a channel ( $2 \mathrm{~mm}$ width, $800 \mu \mathrm{m}$ depth) fabricated by embossing. Fluid inlet tubes were supported with 2-mm thick PDMS slabs, which were connected to the device using a double-sided adhesive layer (3M Command ${ }^{\mathrm{TM}}$ medium picture hanging strips, 17201, www.command.com). Figure 1e shows the laminar flow of two aqueous dye solutions at a rate of $\sim 1 \mathrm{~mL} / \mathrm{min}$ along an embossed channel in a paper microfluidic device. The laminar flow was preserved for flow rates ranging between $0.05-2 \mathrm{~mL} / \mathrm{min}$ without observing any leakage or delamination of the top adhesive layer. We fabricated other microfluidic designs ("T", "U", "Y", see Figure S4, S6, S7) and estimated the Reynolds numbers of water flowing through them to range from 45-1800 (at flow rates ranging from $0.05 \mathrm{~mL} / \mathrm{min}$ to $2 \mathrm{~mL} / \mathrm{min}$, see Supporting Information). All devices showed laminar flow (expected for those Reynolds numbers ${ }^{28}$ ), even when three dye solutions were introduced into the system (Figure S5, Movie_M1 and M2).

Imaging the Channels. To understand whether embossing had a significant influence on the structure of the paper, we compared images of different parts of the devices using scanning electron microscopy (SEM) (Figure 2). Figures 2 b-e shows that the fibers of the paper were stretched in the lateral walls of the channel, since these regions were where the paper was most strained during embossing. The fiber organization in the bottom of the channel is generally retained, i.e. there are no observable structural differences in fiber organization between the bottom and the non-embossed regions of the paper (Figure $2 \mathrm{c}-\mathrm{e}$ ). The depth profile of the embossed channel was also analyzed through contactless surface profilometry (Figure 2a). The roughness of the walls of the channel-where most stress was observed — was $\sim 22 \mu \mathrm{m}$, while the roughness on the bottom of the channel was comparable to regions of the paper device that were not embossed $(\sim 73 \mu \mathrm{m})$. 
Three-Dimensional Microfluidic Devices. We fabricated 3D microfluidic devices on $\mathrm{R}^{\mathrm{F}}$ paper using a two-layer approach (see Figure 3a). To connect the two layers of $\mathrm{R}^{\mathrm{F}}$ paper, we punched holes on the top layer, and through the connecting double-sided tape; these holes allowed the fluid to flow into or out of the bottom layer (Figure 3a, i to iv). To test this design, we passed solutions of dyes in water through the device (Figure 3b-d). As with the 2D microfluidic design, we observed that the fluids moved well across the channel at flow rates ranging from $0.05 \mathrm{~mL} / \mathrm{min}$ to $2 \mathrm{~mL} / \mathrm{min}$ (A video of this device in action is given in the supporting information, Movie_M4). We also ran a 3D microfluidic device using three aqueous solutions: Two on a Y-inlet leading to laminar flow, and the other solution in the cross-channel (Figure S7). We observed that, as the fluid moved along the channel, significant mixing was observed as a reflection of the influence of the channel architecture on the flows and eddies ${ }^{29}$ (see Figure S6, S7, and Movie_M5).

Droplet Generators. Microfluidic droplet generators work by combining streams of immiscible liquids into one channel. ${ }^{30-32}$ When two streams of immiscible liquids, with low Reynolds number, meet in orthogonal channels in a T-junction (with the more rapidly flowing stream in the straight top channel), shear forces break up the more slowly flowing liquid into droplets. Embossed $\mathrm{R}^{\mathrm{F}}$ paper devices are omniphobic with a rough porous surface. To generate droplets, we used a two-inlet " $T$ " shaped device into which we introduced dyed water and dyed hexadecane (Figure 4). Neither the water nor the hexadecane wetted the omniphobic paper. Figure 4a shows a device, with inlets connected; Figure $4 \mathrm{~b}$ shows the same device in use (Movie_M6). We used different flow rates to obtain monodisperse droplets of different sizes and shapes at frequencies between 0.2 and $10 \mathrm{~Hz}$ (Figure 4d-f). The coefficient of variation $(\mathrm{CV})$ of the length of the droplets (the distance between the furthest downstream and upstream points along the interface of a fully detached plug) generated at a 
frequency of $1.25 \mathrm{~Hz}$ was $1.2 \%$. As in other microfluidic devices, ${ }^{33}$ droplet generation with our paper devices follows the simple scaling law (Eq. 1). ${ }^{34,35}$

$$
\frac{L}{w}=1+\alpha \frac{Q_{w a t e r}}{Q_{H D}}
$$

Where $L$ is the length and $w$ is the width of the droplet, $Q_{w a t e r}$ and $Q_{H D}$ are the flow rates of water and hexadecane (HD) respectively, and, $\alpha$ is a constant that depends on the geometry of the junction. A T-inlet device generated using a knife cutter showed similar performance. ${ }^{13}$

Microfluidic Devices made by Cutting and Stacking Omniphobic Paper. We have demonstrated omniphobic paper-based open-channel microfluidic devices that were realized through embossing paper. We also demonstrated that similar devices could be realized by cutting and stacking different paper labels or different layers of paper connected using pre-cut double-sided tape. Figure 5a shows how to make microfluidic devices by stacking a paper label (white labels for laser printers, S-5044, www.uline.com) with the silhouette of the channel cut out on top of a non-patterned label. We made the cut-and-stack paper devices omniphobic by silanization with $\mathrm{R}^{\mathrm{F}} \mathrm{SiCl}_{3}$, and sealed the channel with a layer of transparent adhesive tape, as described before. In comparison to embossing, the cutting and stacking technique has six advantages: i) No molds are needed. The cut patterns can be printed out using a regular printer, or drawn with a pen and a ruler. ii) The process has a higher success rate ( $\sim 98 \%$ of devices work, as compared with $\sim 85 \%$ of embossing). iii) Final construction consists of predictable, uniform, and tunable geometries that can be stack on top of others to configure a final device (Figure 5b). The patterned paper labels and tape serve as the side walls of the channel, enabling the depth of the channel to be readily manipulated, i.e., the depth of the channel is governed by the number of paper layers that are stacked to form the channel. iv) The flat surfaces generated in this procedure make the assembly of the devices 
rapid and easy, using manual procedures. v) The adhesive layers in these devices allows for the direct insertion and sealing of the tubing into the channel, without requiring external adaptors. (Figure 5b,c). vi) Thin microfluidic devices $(\sim 600-\mu \mathrm{m}$ thick devices were fabricated using $150-\mu \mathrm{m}$ thick tape and $220-\mu \mathrm{m}$ thick paper labels) fabricated with this technique can withstand bending and conform to curved surfaces using the adhesive of the bottom label (Figure 5c).

Figure 6 shows one type of 3D paper-based microfluidic device which has two channels crossing each other fabricated by stacking two layers of $\mathrm{R}^{\mathrm{F}}$ paper patterned with channels on top of a layer of non-patterned $\mathrm{R}^{\mathrm{F}}$ paper. The channels are $2 \mathrm{~mm}$ wide and $\sim 80 \mathrm{~mm}$ long. This device has two polyethylene tubes attached to the bottom layer of $\mathrm{R}^{\mathrm{F}}$ paper to deliver gases, through the paper, into the channels (Figure 6a). Two aqueous solutions of $\mathrm{pH}$ indicator (yellow_-Phenol Red; dark brown-Bromophenol Blue sodium salt) were introduced into the channels through the two inlets located on the top left part of the device (Figure 6b). When no gas is delivered into the channels, the color of the liquid at the outlets indicates that the device enables streams of fluid to cross one another multiple times without mixing (Figure 6c). When we deliver $\mathrm{HCl}(\mathrm{g})$ and $\mathrm{NH}_{3}(\mathrm{~g})$ through polyethylene tubes attached to the back of the device, the gases diffused through the bottom $\mathrm{R}^{\mathrm{F}}$ paper layer in the channels containing the indicator solution. The gases dissolve in the solution flowing through the channel, change the $\mathrm{pH}$ of the solution, and are visualized by a color change in the indicator dyes (Figure 6d). The yellow-Phenol Red solution changes to purple under basic conditions, and the dark brown-Bromophenol Blue sodium salt solution changes to orange on exposure to acidic conditions. The high gas permeability of $\mathrm{R}^{\mathrm{F}}$ paper provides a simple way to introduce a gas to a liquid (to capture components of the gas or as part of a sensor) without having a large exposed surface of liquid. The gas impermeable tape 
that is used to connect different layers prevents exposure of large areas of the flowing fluid to permeating gases, allowing the delivery to be targeted or localized.

Microfluidic Displays. After demonstrating a working gas-permeable 3D microfluidic device made by the cut-and-stack approach, we fabricated another microfluidic device with three letters "G", "M", and "W" connected via bottom channels following the cutting and stacking process described previously (Figure 5 and 6). It has one inlet and one outlet on the top right part of "G" and "W", respectively, as shown in Figure 7a. The inlet and outlet in this device are both on the back of the device. We used Bromothymol Blue solution, a universal indicator ( $\mathrm{pH}$ sensing range 6.0-7.6, displaying yellow, green, and blue in acidic, neutral, and alkaline solutions, respectively), to demonstrate the possibility to induce sequential changes in the $\mathrm{pH}$ of a solution flowing through a microchannel made of $\mathrm{R}^{\mathrm{F}}$ paper by taking advantage of the gas permeability of this paper device. The three letters displayed in Figure 7a were initially green with the solution flowing from "G" to "W" along the direction of the arrow. A vial containing a solution of $15 \% \mathrm{NH}_{4} \mathrm{OH}\left(\mathrm{NH}_{3}\right.$ source) was connected to the back of the device via a 1/32" diameter Teflon tube. We inserted the end of the tube into a PDMS slab for support and attached the tube to the non-patterned bottom layer of the device using a ring of double-sided adhesive tape. The $\mathrm{NH}_{3}(\mathrm{~g})$ source was connected to the "M" on the back of the device (bottom left of "M", as indicated by an arrow in Figure 7b), resulting in a colorimetric change of " $\mathrm{M}$ " and "W" from green to blue. The color of letter "G" remained green, indicating that $\mathrm{NH}_{3}$ did not diffuse into the letter "G" under fluid flow. After the exposure of "M" to $\mathrm{NH}_{3}(\mathrm{~g})$, we exposed the letter "W" (arrow in Figure 7c) to $\mathrm{HCl}$ (g) coming through the bottom paper layer in an analogous way to what was done for $\mathrm{NH}_{3}$ (g). A vial containing concentrated $\mathrm{HCl}$ (fuming $\mathrm{HCl}$ ) was used as our acidic gas source. The acidic gas, $\mathrm{HCl}(\mathrm{g})$, neutralized the ammonium hydroxide present in the solution of indicator and split the fluid flowing through the "W" into two $\mathrm{pH}$ regions where the solution again 
turned yellow on the side of the microfluidic channel exposed to the acid while the other half remained blue (Figure 7c). We used this effect to demonstrate that the gas-permeability of a microchannel can be controlled by partially covering the width of the channel with a gasimpermeable layer, like tape, therefore limiting the ability for a gas to be introduced to the whole channel. A closer look at the inlet and outlet (Figure 7a,b) of each letter reveals a square dark spot in the background except for the inlet of letter "W" which has a triangular dark spot. This darkened spots are the channels that connect the letters except that for letter "W" the channel has been partially blocked with tape to allow gas diffusion to only occur through half of the channel (the unblocked triangle). When we attached the $\mathrm{HCl}$ inlet tube under this channel, only half of the channel changed color from blue to yellow. The acid (yellow) and basic (blue) solutions, as expected, did not mix under laminar flow. After the fluid flow was stopped, the yellow and blue solutions gradually mixed due to diffusion (Figure 7d). Figure 7e-h show a similar channel filled with a red aqueous solution and blue dye in hexadecane demonstrating the omniphobicity of the paper channel.

96-zone Plate. We fabricated 96-zone plates by mechanically embossing Whatman \#5 chromatography paper between two ABS dies and rendering the surfaces omniphobic by silanization with $\mathrm{R}^{\mathrm{F}} \mathrm{SiCl}_{3}$ (see Figure S2). Embossed wells with a diameter of $12 \mathrm{~mm}$ and a depth of $2 \mathrm{~mm}$ could hold aqueous solutions with a volume of up to $100 \mu \mathrm{L}$. We similarly fabricated well-plates of tunable depth and diameter through the cut-and-stack method. The depth of the well was controlled by the number of layers used to make the device, while the diameter of the wells was determined by the size of holes made on the paper (Figure S3). 


\section{Conclusions}

A two-step process_-embossing or cutting, treatment with perfluorinated $\mathrm{R}^{\mathrm{F}} \mathrm{SiCl}_{3}$ as a vapor, followed by assembly_makes it possible to fabricate open channel microfluidic devices on paper rapidly. Dies for embossing were fabricated in $\mathrm{ABS}$ using a 3D printer; they could be used multiple times (over 1000) with no damage or degradation in performance. Fluid flow behavior in these paper-based microfluidic devices is similar to that observed in PDMS-based open channel devices. We observed laminar flow, droplet generation through shearing in a T-device, and 3D flow in a multi-layer device made by placing different layers of embossed paper on top of each other and connected them using double-sided adhesive tape. By connecting different layers through pre-cut hole, and covering the top with a layer of transparent adhesive tape, we created 3D microfluidic devices in which fluids could past one or another in different layers without mixing. In principle, the gas-permeable devices reported here can be applied to any liquid- or solid-gas physisorption process or chemical reaction with a broad range of applications in analysis, environmental monitoring, infochemistry, particle synthesis, and many others.

The cut-and-stack method is ideal for fabrication of reconfigurable 3D devices since the depth of the channels can be controlled by stacking layers of paper or using paper with different thicknesses. Adhesive and bendable microfluidic devices (as thin as $600 \mu \mathrm{m}$ ) were fabricated using paper labels and tape. Since paper can be folded, cut-and-stack method can be combined with origami to build complex fluid transport systems or devices. Although embossing and the engraving techniques have their advantages, the versatility of the cut-andstack method allows one to build more robust devices in a reliable manner using simple tools. 
Fig. 1
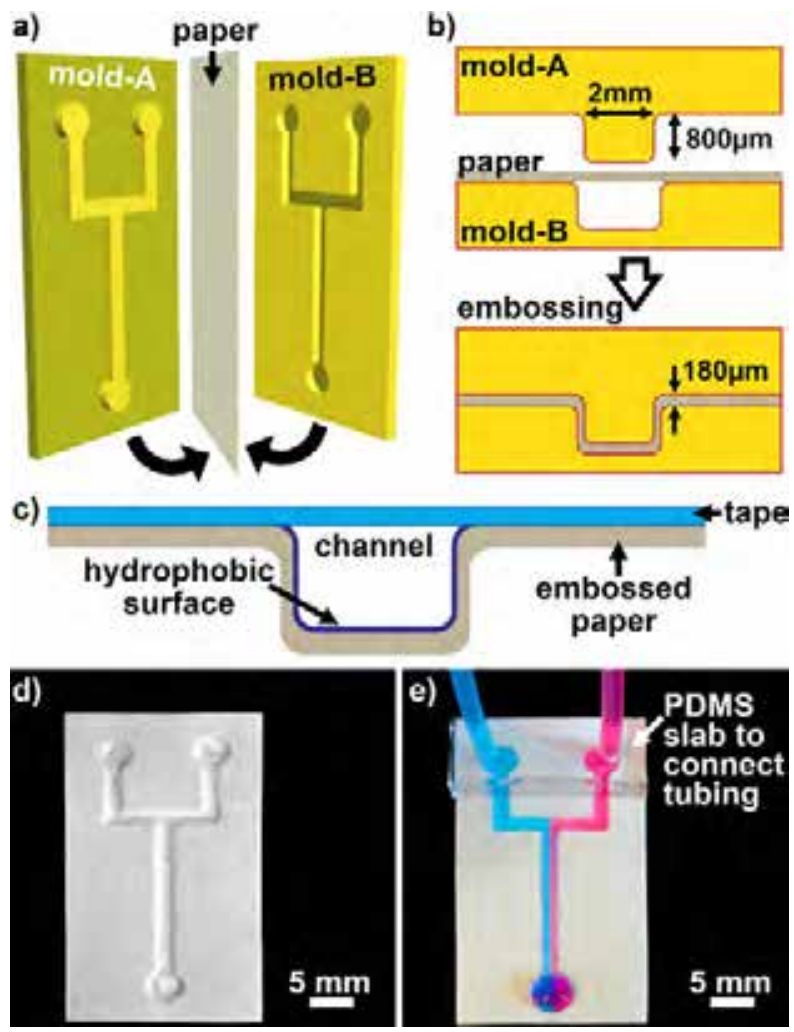

Figure 1. Laminar flow in an open-channel microfluidic device constructed by embossing channels on Whatman \#1 filter paper. a) The embossing process in which a sheet of paper is placed between two plastic molds and pressed-for dimensions, see supporting information Figure S1. b) Cross-section schematic diagram of the embossing process. c) Cross-sectional view of the finished channel with the different parts highlighted. d) A picture of a two inlet finished device before connecting the fluid inlet tubes. The inlet tubes are supported by a small piece of PDMS elastomer and attached to the inlets using double-sided tape. e) A picture of two streams of aqueous dye solution flowing through the microfluidic channel. 
Fig. 2

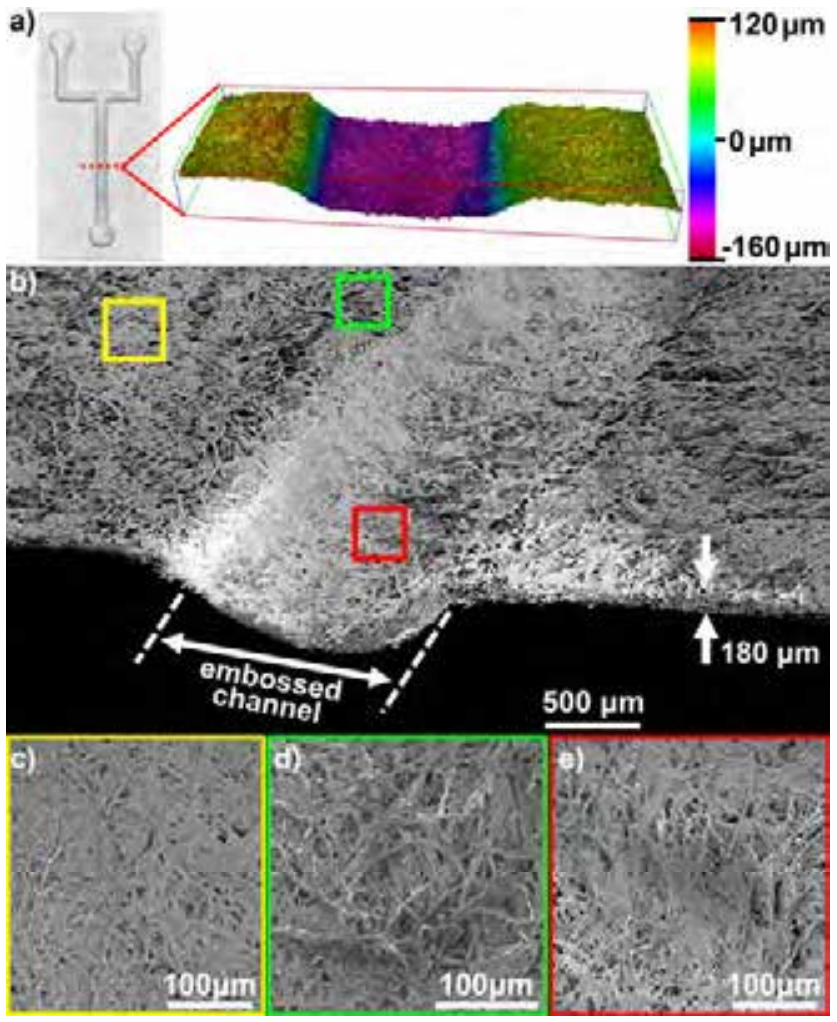

Figure 2. Physical characterization of the embossed microfluidic devices. a) Cross-section image of the embossed channel of our microfluidic devices. The image was obtained from a ready-to-use paper device analyzed using a contactless surface profilometer. As expected, there is a sharp decline in height on the shoulders of the channel but the roughness of the bottom of the channel is comparable to that of the non-embossed regions of the paper.

b) SEM image of the embossed channel made from Whatman \#1 filter paper shown in a).

c) Highlight of a non-embossed part of the paper, distant from the embossed channel;

d) A non-embossed region on the shoulder of the embossed channel; e) A region of the paper inside the channel. 
Fig. 3

a)

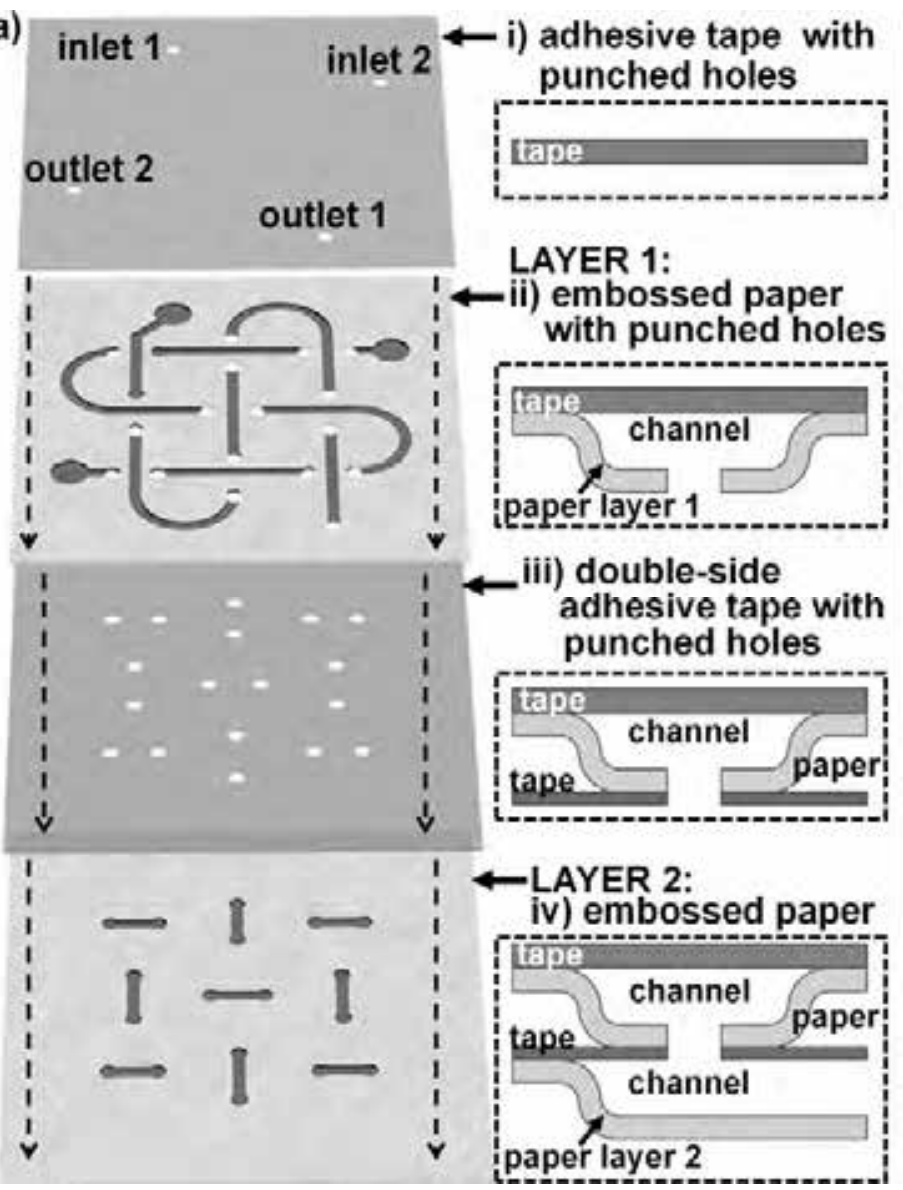

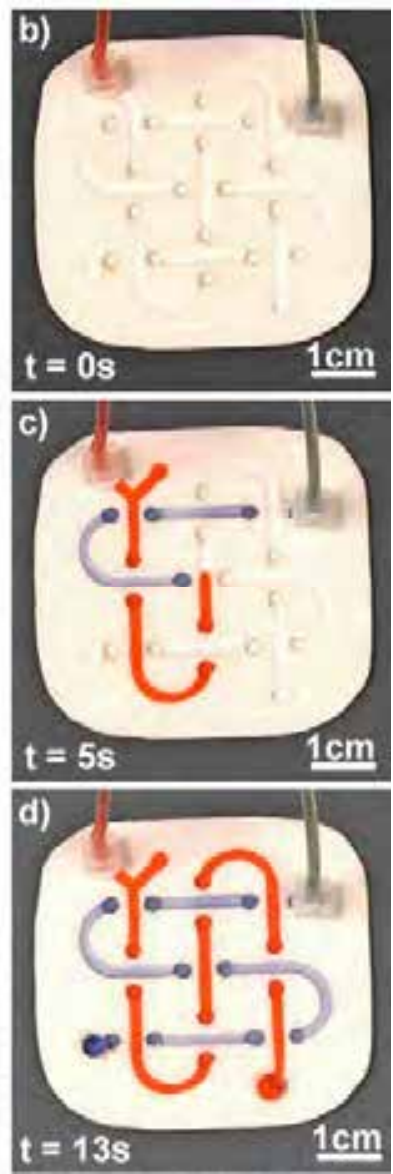

Figure 3. a) Schematic representation of components used to fabricate the $3 \mathrm{D}$ embossed microfluidic devices. i) Transparent single-sided tape (grey) with pre-cut holes for the fluid inlets and outlet, ii) Layer 1; top layer of channels embossed in $\mathrm{R}^{\mathrm{F}}$ paper, with holes to allow liquid to flow under or into channels on the top paper layer. The holes are formed after embossing using a hand-held office punch or by using a laser cutter. iii) double-sided adhesive tape with pre-cut holes used to connect the top and bottom channel systems (layers 1 and 2). Alignment to the two layers is critical. iv) Layer 2; bottom layer of embossed paper channels that allow liquids to flow underneath the top layer. b) A finished 3D microfluidic device with two inlets, attached using double-sided tape $(t=0 \mathrm{~s})$. c) The device during a run ( $\mathrm{t}=5 \mathrm{~s}$, see Movie_M4) showing back flow at the "Y" intersection then flowing through channels in both levels. d) After flowing $\sim 2 \mathrm{~mL}$ of liquid through the device, two drops (blue and red) can be seen accumulating on the outlet. 
Fig. 4
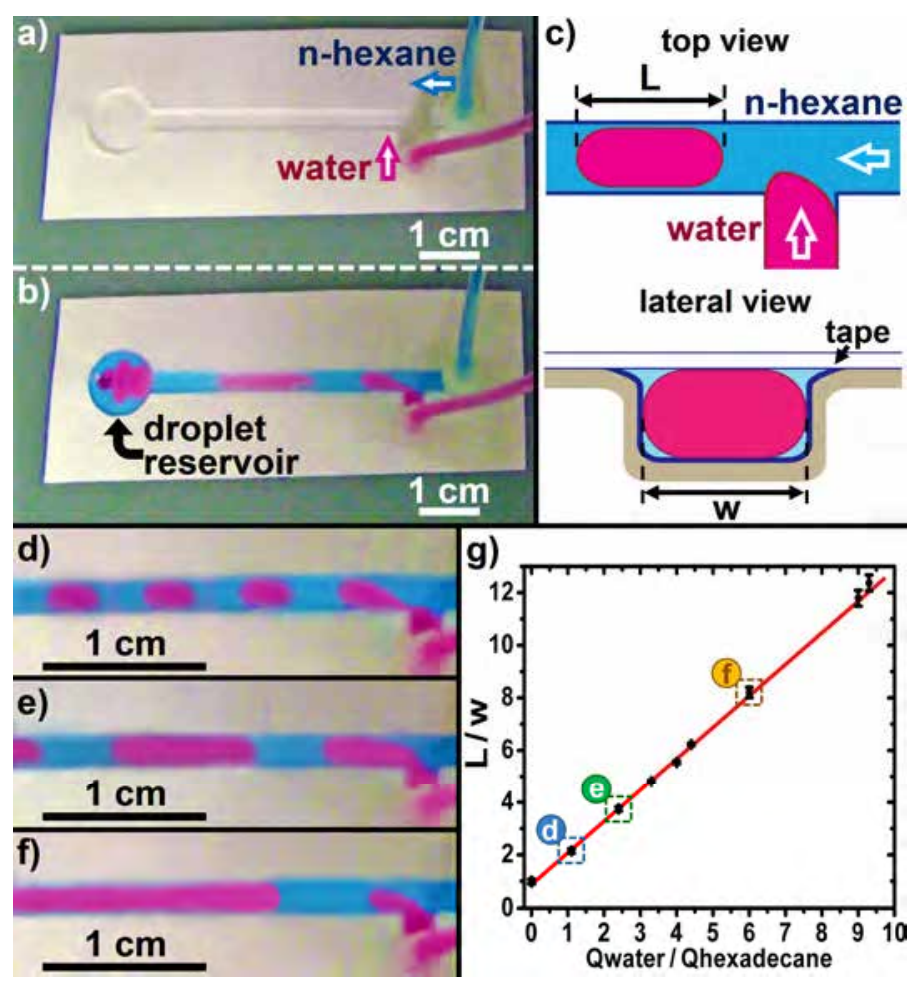

Figure 4. Droplet generator based on a "T"-shaped microfluidic device: a) Embossed

T-junction microfluidic device with the inlet tubes connected using PDMS slabs. b) Droplet generator in use. c) Schematic illustration of the geometry of the droplets inside the channel. (d-f) Droplet generator working at $9.5 \mathrm{~Hz}$ (d), $3.4 \mathrm{~Hz}(\mathrm{e})$, and $0.5 \mathrm{~Hz}$ (f). g) Linear dependence on the flow rates of $\mathrm{L} / \mathrm{w}$ in this system. Highlighted data correspond to the cases shown in panels d-f. 
Fig. 5

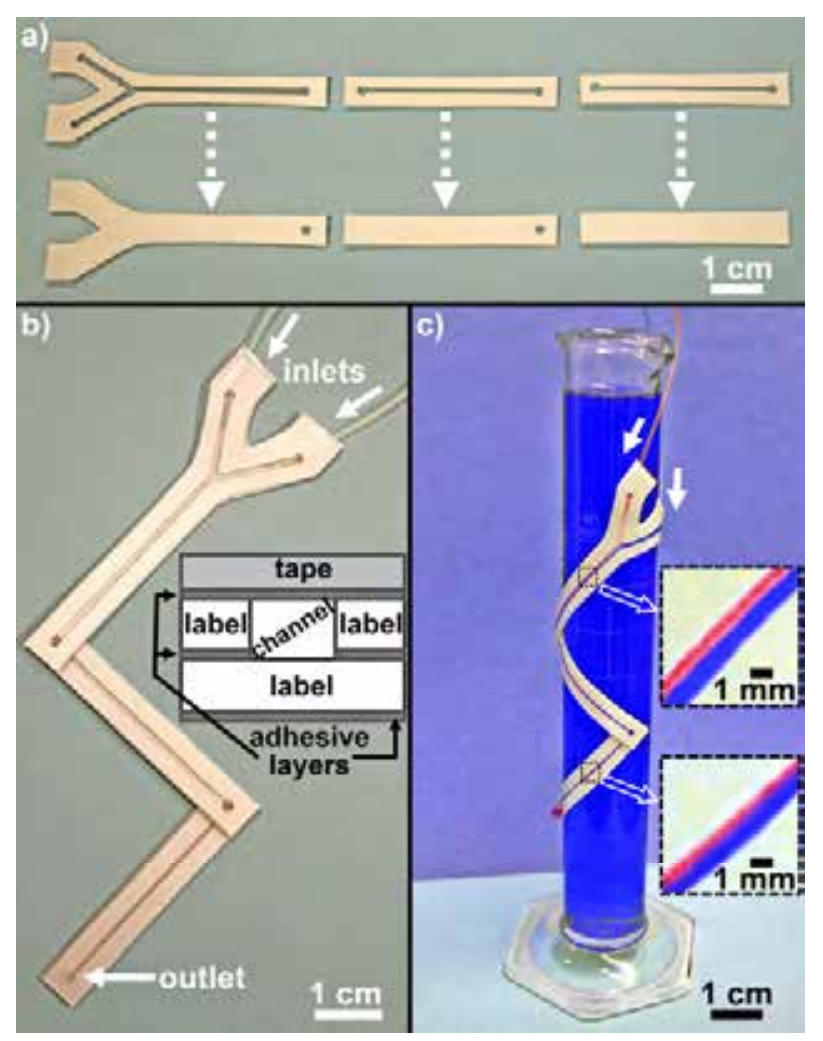

Figure 5. a) Paper labels trimmed with a scalpel. The labels patterned with the outline of the channels (top) were stuck on top of the labels that will serve as the bottom of the channel and the ensemble was silanized. b) Paper device formed after sealing the channels by adhering a layer of transparent tape on top of the RF paper device (total thickness $\sim 600 \mu \mathrm{m}$ ) and stuck the different sections of the device together using the back adhesive layer of the bottom paper label. The tubing connecting this device with a syringe pump can be secured by using the adhesive layer of the paper label at the top. This device admits to be reconfigured by changing the angle between its different sections. c) Bendable microfluidic device conforming to the curved surface of a graduated cylinder containing an aqueous solution of Methylene Blue. This pressure-driven paper microfluidic device exhibits laminar flow when two miscible aqueous phases, each labeled with a different water-soluble dye $(0.05 \%$ solutions of Methylene Blue or Congo Red in water), were pumped through a Y-junction at a flow rate of $100 \mu \mathrm{L} / \mathrm{min}$ (Reynolds number $\mathrm{Re}=80$ ) 
Fig. 6

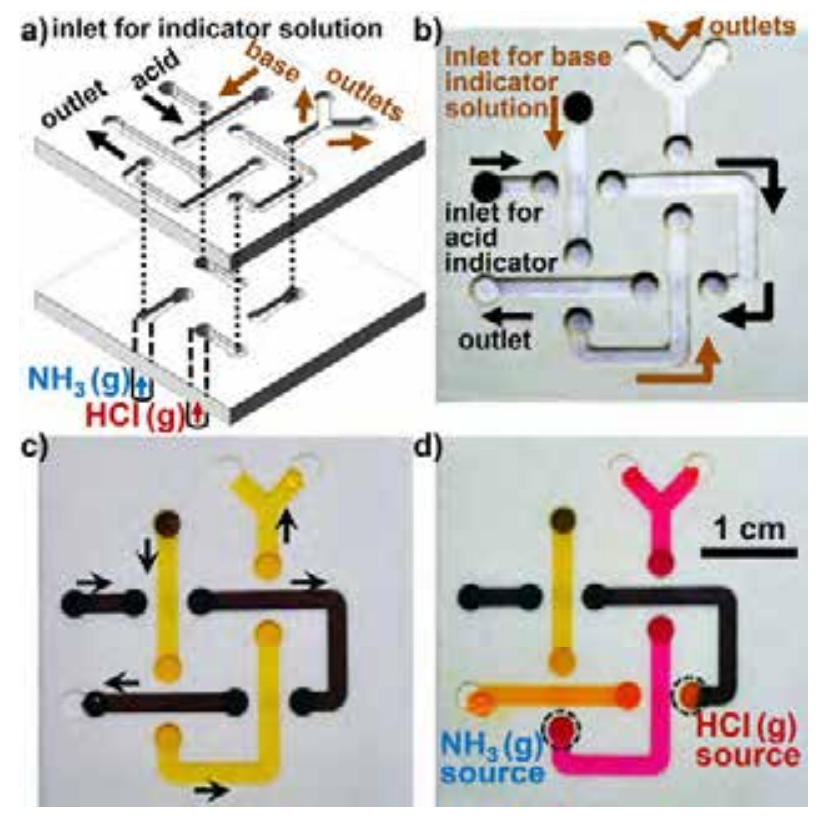

Figure 6. a) Schematic illustration of a paper-based 3D microfluidic device fabricated the cut-and-stack method - for more details, see supporting information Figure S8. The device allows multiple streams of fluid (acid and base) to cross each other without mixing. Gas sources are connected to the back of the channels; arrows indicate the directions of fluid and gas flow through the device. Note: the top layer of polymer tape and bottom layer of uncut paper are not shown to highlight the fluidic channels. b) Photographs the 3D microfluidic device. Fluid inlets are connected to the underside of the device (indicated by a darker coloration in the channel). c) Flowing phenol red (yellow) and bromophenol blue sodium salt (brown) $\mathrm{pH}$-indicator solutions through the device channels. d) The yellow indicator changes color to red when exposed to basic gas $\left(\mathrm{NH}_{3}\right)$ and the dark brown solution changes color to orange under acidic conditions. Gas inlets attachment locations are indicated by black dotted circles in d). 
Fig. 7
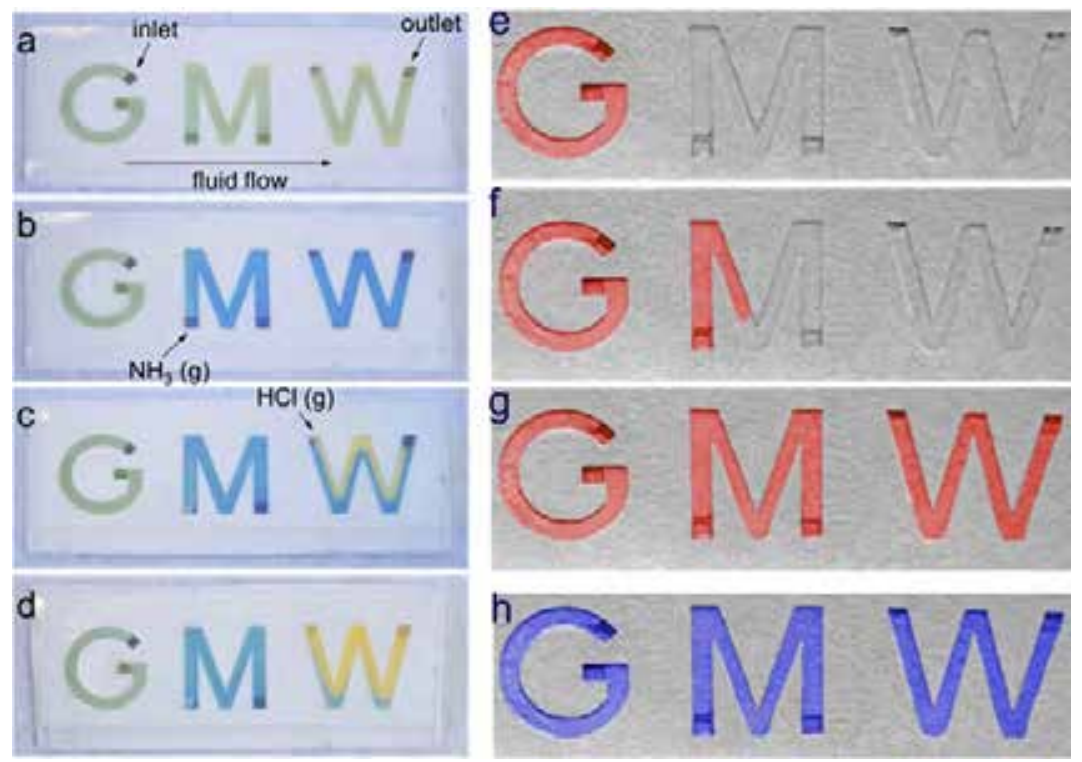

Figure 7. A cut-and-stack 3D microfluidic device with three letters (“G”, "M", and "W") making up the channels was made. a) The device with bromothymol blue solution only. The solution flowed from "G" to "W" with the inlet and outlet as indicated. b) The starting point of "M" (where the arrow points to) was exposed to $\mathrm{NH}_{3}$ (g) from the back of the device. The color of "M" and "W" changed from green to blue. c) The triangular part in "W" (indicated by the arrow) was exposed to $\mathrm{HCl}(\mathrm{g})$. The color of "W" changed partially from blue to yellow. d) The solution was stopped and the yellow and blue solutions, as expected, diffused and mixed in "W" as expected. (e-g) show an aqueous red solution at different times in the channel filling process. h) Show the same channel filled with a blue dye in hexadecane to demonstrate the omniphobicity of the paper channel. 


\section{ASSOCIATED CONTENT}

Supporting Information: More detailed details on the dimensions of the dies, additional devices and videos of the running experiments are available in the supporting information. This material is available free of charge via the Internet at http://pubs.acs.org or from the website of the Whitesides group (http://gmwgroup.harvard.edu/pubs)

\section{AUTHOR INFORMATION}

\section{Corresponding Authors:}

Prof. George M. Whitesides

Department of Chemistry and Chemical Biology, Harvard University, 12 Oxford Street, Cambridge, MA 02138, USA.

*E-mail: gwhitesides@gmwgroup.harvard.edu

Author Contributions: G.M.W., M.M.T., R.V.M., and X. L. conceptualized the project. M.M.T., R.V.M., X.L., and M.B.J.A. developed techniques for embossing. J.B., M.M.T. and R.V.M imaged the devices, M.M.T., W.L., J.B., and R.V.M developed the cut-and-stack methodology. R.V.M., M.M.T., and W.L. characterized the performance of the devices. G.M.W. advised and oversaw the research. All authors contributed to the writing of the manuscript. R.V.M. and M.M.T. contributed equally to this work. 


\section{Funding Sources:}

Bill and Melinda Gates Foundation (award 51308).

Department of Energy (DOE, DESC0000989).

Materials Research Science and Engineering Center (MRSEC, award DMR-0820484).

European Commission (Marie Curie IOF-275148).

National Science Foundation (award PHY-0646094).

Note: The authors declare no competing financial interest.

\section{ACKNOWLEDGMENTS}

This research was supported by the Bill and Melinda Gates Foundation under award 51308. M.M.T. acknowledges support from a Nanoscale Science and Engineering Centre fellowship at Harvard University and a sub-contract from a Department of Energy (DESC0000989) award to Northwestern University for salary support. R.V.M. acknowledges funding by the FP7 People program under the project Marie Curie IOF-275148.

J. B. acknowledges salary support from the Materials Research Science and Engineering Center under MRSEC award DMR-0820484. This work was performed in part using the facilities of the Center for Nanoscale Systems (CNS), a member of the National Nanotechnology Infrastructure Network (NNIN), which is supported by the National Science Foundation under NSF award PHY-0646094. CNS is part of the Faculty of Arts and Sciences at Harvard University. 


\section{TABLE OF CONTENTS}

Omniphobic " $R$ F" paper is used to fabricate open-channel microfluidic systems by embossing or "cut-and-stack" methods. Microfluidic devices made of $\mathrm{R}^{\mathrm{F}}$ paper are mechanically flexible and highly permeable to gases, which allows processes not possible in microfluidic devices made of PDMS or other non-porous polymers.

Keywords: Microfluidics, Omniphobic paper, Embossing, Cut-and-stack, Paper-based Microfluidics.

Martin M. Thuo, Ramses V. Martinez, Wenjie Lan, Xinyu Liu, Jabulani Barber, Manza B. J. Atkinson, Dineth Bandarage, Jean-Francis Bloch, and George M. Whitesides

Fabrication of Low-cost Paper-based Microfluidic Devices by Embossing or Cut-and-Stack Methods.

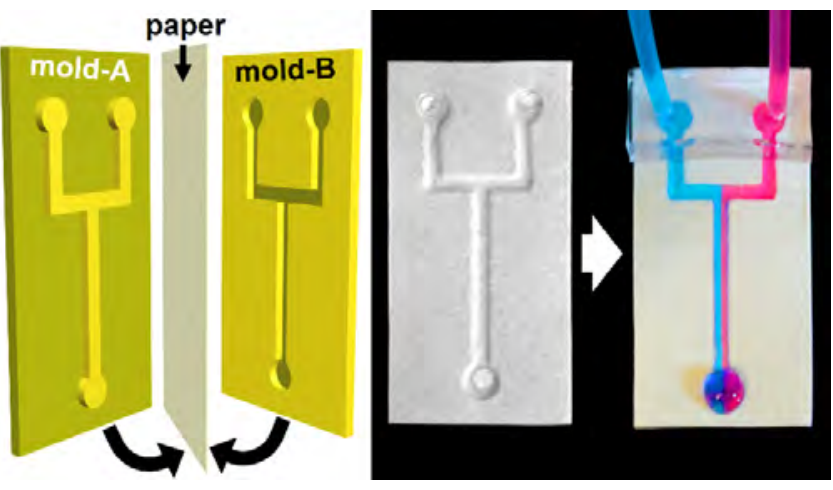




\section{REFERENCES}

1. Chin, C. D.; Laksanasopin, T.; Cheung, Y. K.; Steinmiller, D.; Linder, V.; Parsa, H.; Wang, J.; Moore, H.; Rouse, R.; Umviligihozo, G. Nat. Med. 2011, 17, 1015-19.

2. Neethirajan, S.; Kobayashi, I.; Nakajima, M.; Wu, D.; Nandagopal, S.; Lin, F. Lab Chip 2011, 11, 1574-86.

3. Jokerst, J. C.; Emory, J. M.; Henry, C. S. Analyst 2012, 137, 24-34.

4. Ellerbee, A. K.; Phillips, S. T.; Siegel, A. C.; Mirica, K. A.; Martinez, A. W.; Striehl, P.; Jain, N.; Prentiss, M.; Whitesides, G. M. Anal. Chem. 2009, 81, 8447-52.

5. Martinez, A. W.; Phillips, S. T.; Whitesides, G. M.; Carrilho, E. Anal. Chem. 2009, $82,3-10$.

6. Nie, Z.; Nijhuis, C. A.; Gong, J.; Chen, X.; Kumachev, A.; Martinez, A. W.; Narovlyansky, M.; Whitesides, G. M. Lab Chip 2010, 10, 477-83.

7. Dungchai, W.; Chailapakul, O.; Henry, C. S. Anal. Chem. 2009, 81, 5821-26.

8. Mao, X.; Huang, T. J. Lab Chip 2012, 12, 1412-16.

9. Schilling, K. M.; Lepore, A. L.; Kurian, J. A.; Martinez, A. W. Anal. Chem. 2012, 84, 1579-85.

10. Carrilho, E.; Martinez, A. W.; Whitesides, G. M. Anal. Chem. 2009, 81, 7091-95.

11. Lu, Y.; Shi, W.; Jiang, L.; Qin, J.; Lin, B. Electrophoresis 2009, 30, 1497-500.

12. Bras, J.; Sadocco, P.; Belgacem, M. N.; Dufresne, A.; Thielemans, W. Mater. Chem. Phys. 2010, 120, 438-45.

13. Glavan, A. C.; Martinez, R. V.; Maxwell, E. J.; Subramaniam, A. B.; Nunes, R. M.; Soh, S.; Whitesides, G. M. Lab Chip 2013, 13, 2922-30.

14. Glavan, A. C.; Martinez, R. V.; Subramaniam, A. B.; Yoon, H. J.; Nunes, R.; Lange, H.; Thuo, M. M.; Whitesides, G. M. Adv. Funct. Mater. 2014, 24, 60-70. 
15. Roper, M. G.; Shackman, J. G.; Dahlgren, G. M.; Kennedy, R. T. Anal. Chem. 2003, $75,4711-17$.

16. Plouffe, B. D.; Radisic, M.; Murthy, S. K. Lab Chip 2008, 8, 462-72.

17. Wu, Z.; Willing, B.; Bjerketorp, J.; Jansson, J. K.; Hjort, K. Lab Chip 2009, 9, 1193 99.

18. Zhu, Y.; Chen, H.; Du, G.-S.; Fang, Q. Lab Chip 2012, 12, 4350-54.

19. Li, L.; Ismagilov, R. F. Biophysics 2010, 39.

20. Xie, J.; Miao, Y.; Shih, J.; Tai, Y.-C.; Lee, T. D. Anal. Chem. 2005, 77, 6947-53.

21. Li, W.; Greener, J.; Voicu, D.; Kumacheva, E. Lab Chip 2009, 9, 2715-21.

22. Abate, A. R.; Kutsovsky, M.; Seiffert, S.; Windbergs, M.; Pinto, L. F.; Rotem, A.; Utada, A. S.; Weitz, D. A. Adv. Mater. 2011, 23, 1757-60.

23. Bhagat, A. A. S.; Hou, H. W.; Li, L. D.; Lim, C. T.; Han, J. Lab Chip 2011, 11, 187078.

24. Martinez, R. V.; Branch, J. L.; Fish, C. R.; Jin, L.; Shepherd, R. F.; Nunes, R.; Suo, Z.; Whitesides, G. M. Adv. Mater. 2013, 25, 205-12.

25. Novak, R.; Ranu, N.; Mathies, R. A. Lab Chip 2013, 13, 1468-71.

26. Kunnari, V.; Salminen, K.; Oksanen, A. Pap. Puu-Pap. Tim. 2007, 89, 46-49.

27. Qin, D.; Xia, Y.; Whitesides, G. M. Nat. Protoc. 2010, 5, 491-502.

28. Hetsroni, G.; Mosyak, A.; Pogrebnyak, E.; Yarin, L. Int. J. Heat Mass Tran. 2005, 48, 1982-98.

29. Naher, S.; Orpen, D.; Brabazon, D.; Morshed, M. M. Adv. Mater. Res. 2010, 83, 93139.

30. Leshansky, A.; Afkhami, S.; Jullien, M.-C.; Tabeling, P. Phys. Rev. Lett. 2012, 108, 264502. 
31. Weaver, J. A.; Melin, J.; Stark, D.; Quake, S. R.; Horowitz, M. A. Nat. Phys. 2010, 6, 218-23.

32. Suh, Y. K.; Kang, S. Micromachines 2010, 1, 82-111.

33. Nisisako, T.; Torii, T.; Higuchi, T. Lab Chip 2002, 2, 24-26.

34. Garstecki, P.; Fuerstman, M. J.; Stone, H. A.; Whitesides, G. M. Lab Chip 2006, 6, $437-46$.

35. Xu, J.; Li, S.; Tan, J.; Luo, G. Microfluid. Nanofluid. 2008, 5, 711-17. 